\title{
Measurements of Raman scattering by electrons in metals: The effects of electron-phonon coupling
}

\author{
Yu. S. Ponosov ${ }^{1}$ and S. V. Streltsov ${ }^{1,2}$ \\ ${ }^{1}$ Institute of Metal Physics UD RAS, 620990, S. Kovalevskaya str. 18, Ekaterinburg, Russia \\ ${ }^{2}$ Ural Federal University, Mira str. 19, Ekaterinburg, Russia
}

\begin{abstract}
We report the first systematic measurements of the Raman scattering by electrons in elemental metals of $\mathrm{Al}, \mathrm{Mo}, \mathrm{Nb}, \mathrm{Os}, \mathrm{Pb}, \mathrm{Re}, \mathrm{Ta}, \mathrm{Ti}, \mathrm{V}, \mathrm{W}$ and metallic compound La $B_{6}$. Experimental spectra are modelled on the base of the band structures, calculated within the density functional theory, taking properly into account the effects of electron-phonon scattering. The agreement between our measured and calculated spectra is excellent for the variety of metals, thus providing the information on the electron self-energies and estimates for the electron-phonon coupling constants and temperature-dependent relaxation rates. The method can be applied for other metallic materials to evaluate an electron-phonon coupling as an alternative to the transport and optical measurements.
\end{abstract}

PACS numbers: 78.30.-j, 74.25.nd, 74.70.Ad, 71.38.Cn

After the discovery of high-temperature superconductors (HTSC) the electronic Raman scattering (ERS) has been widely used to study electronic excitations both in superconducting and normal state of a variety of compounds [1]. The ERS provides information on the twoparticle correlation function, which is in close correspondence to transport properties. In addition to studies of the magnitude and symmetry properties of the superconducting gap, many investigations of the ERS have been devoted to the problem of many-body interactions. Since clear understanding of the electron self-energy origin may shed light on the leading mechanism of unusual superconductivity, the ERS has become an important complementary tool for the electron-dynamics investigations [1]. Thus, the observation of a flat, nearly frequency-independent, electronic Raman response in the normal state of cuprates [2 [6] has become the basis of a marginal Fermi liquid concept [7]. Models, incorporating different electron interactions, like nested Fermi liquid [8] or antiferromagnetic spin fluctuations [9, 10], have been proposed to explain the unusual ERS spectra of HTSC. The phonon origin of the electron selfenergy in cuprates is still debated [11]. Broad Raman spectra very similar to a marginal Fermi liquid behavior have been simulated for strongly coupled electronphonon systems [12 14]. However, no experimental evidence for such behavior has been presented in early studies of the strongly-coupled conventional superconductors. Moreover, no normal-state spectra have been measured for $\mathrm{Nb}, 15$, , 16] and the origin of the broad continuum in A-15 compounds, which is evolved in superconducting state, is still not fully understood [15 18]. Thus, the investigations of the ERS spectra in the normal state of conventional metals and compounds, where an electronphonon interaction seems to be the main contribution to the electron self-energy, and of their evolution upon the change of coupling strength and temperature are actually lacking.

The first unsuccessful attempts to observe the Raman scattering from electronic excitations in conventional metals were performed more than sixty years ago 19,20$]$. The following calculation of the Raman response 21] has shown that the sensitivity in these measurements was by 5 orders of magnitude low. In spite of the substantial advances of the Raman technique nowadays, the widespread opinion consist in that the measurements of the Raman effect from electrons in conventional metals is a difficult challenge. It is because the charge-density fluctuations are largely screened by conducting electrons and the $\mathrm{q} \rightarrow 0$ limit, usually used in the experiment treatment, implies the smallness of electronic scattering cross section which is proportional to $q^{2}$ at low frequencies.

Most of elemental metals, excluding some alkali ones, have the anisotropic multisheeted Fermi surfaces. This leads to nonvanishing unscreened low-frequency scattering [22]. The increase of the effective q-vector because of the strong absorption at a metallic surface [23] (up to $2 \times 10^{6} \mathrm{~cm}^{-1}$ for the exciting laser energies $\omega_{i}$ in the visible range) also enhances the scattering intensity 21, 24]. With electron velocities $v_{f}$ being as high as $10^{8} \mathrm{~cm} / \mathrm{sec}$, the electronic excitation's energies $\omega=q v_{f}$ in the Raman spectra can spread up to $1000 \mathrm{~cm}^{-1}$. Such large $q v_{f}$ values can not be neglected when treating the ERS in metals. The found resonance effects [25] are another factors assisting the observation ERS spectra in metals.

In this report we present the first systematic investigations of the temperature-dependent ERS spectra in the normal state of elemental metals and metallic compounds. The obtained spectra have been compared with those simulated based on the band structures, calculated within the density functional theory, taking into account the electron-phonon interaction. The groups of metals with weak and strong electron-phonon interaction were found to have strongly distinct Raman spectra and their temperature dependences. The results of the measurements and their analysis provided independent estimations of the electron-phonon coupling constants and electron relaxation rates. 
For temperature-dependent measurements (10-300K), electropolished plates of single crystals (residual resistance ratio $\geq 50$ ) were placed into an Oxford optical cryostat. The Raman spectra were excited by low-power laser radiation (up to $3 \mathrm{~mW}$ ) at wavelengths of $514 \mathrm{~nm}$ and 633 $\mathrm{nm}$, and they were recorded by a single-stage Renishaw microscope spectrometer providing a focal spot on the samples of $2 \div 10 \mu \mathrm{m}$ diameter. The represented spectra were corrected for the Bose factor, spectral response of the spectrometer, and transmission and absorption coefficients in the frequency range of the scattered light.

The cross section for the ERS by the intraband excitations is determined via integrating the electron susceptibility $\chi(q, \omega)$ over the distribution of excited wave vectors $U^{2}(q)$ 24, 26]:

$$
\frac{d^{2} \sigma}{d \omega d \Omega} \propto \frac{1}{1-\exp ^{-\hbar \omega / k T}} \int_{0}^{\infty} d q \cdot U^{2}(q) \cdot \chi(q, \omega)
$$

When the interaction between electrons is taken into account, the susceptibility $\chi(q, \omega)$ can be written in the form of the integral of the imaginary part of the qdependent polarization operator over the Fermi surface $[24,26]$ :

$$
\begin{aligned}
& \chi_{\alpha, \beta}(q, \omega)=\oint \frac{d s_{f}}{v_{f}}\left|\gamma_{\alpha, \beta}(k)\right|^{2} \int_{-\infty}^{\infty} d \epsilon[f(\epsilon)-f(\epsilon+\omega)] \\
& \times \Im \frac{1}{\omega-q v_{z}-\Sigma^{\prime}(\epsilon+\omega)+\Sigma^{\prime}(\epsilon)-i\left[\Sigma^{\prime \prime}(\epsilon+\omega)+\Sigma^{\prime \prime}(\epsilon)\right]}(2)
\end{aligned}
$$

The similar formalism has been previously used in the calculations of the nonadiabatic effects in the phonon self-energy 27] and the ERS spectra in the $\mathrm{q} \rightarrow 0$ limit 12 14]. Here, $f(\varepsilon)$ is the Fermi function, and $z$ denotes the normal to the sample surface. The electron velocities on the Fermi surface have been obtained within the bandstructure calculations using the linearized muffin-tin orbital method (TB-LMTO-ASA) 28]) in the local density approximation. Integration over the Fermi surface was performed with a fine mesh of $125,000 \mathrm{k}$-points in the full Brillouin zone.The retarded and advanced quasi-particle electron self-energies $\Sigma(\epsilon)$ and $\Sigma(\epsilon+\omega)$ determine the electron spectrum renormalization near the Fermi level due to different interactions. In the case of electronphonon scattering, their real and imaginary parts are 29]

$$
\begin{array}{r}
\Sigma^{\prime}(\omega)=\int d \Omega \alpha^{2} F(\Omega) \\
\times \Re\left[\psi\left(\frac{1}{2}+i \frac{\omega+\Omega}{2 T}\right)-\psi\left(\frac{1}{2}+i \frac{\omega-\Omega}{2 T}\right)\right] \\
\Sigma^{\prime \prime}(\omega)=\pi \int d \Omega \alpha^{2} F(\Omega) \\
\times\left[2 n_{B}(\Omega)-f(\omega-\Omega)+f(\omega+\Omega)+1\right]+\nu
\end{array}
$$

The impurity relaxation rate $\nu$ was taken equal 10 $\mathrm{cm}^{-1}$ in all calculations (this value is the upper limit for used pure samples), $\Psi$ is the digamma function, $\Omega$ is the phonon energy, $\alpha^{2} F(\Omega)$ is the Eliashberg spectral function for the electron-phonon interaction, and $n_{\mathrm{B}}(\Omega)$ is the Bose function. The literature data on the phonon densities of states $F(\Omega)$ 30] and optical constants $\mathrm{n}$ and $\mathrm{k}$ [31] for the calculation of the function $U^{2}(\mathrm{q})$ were used. Following [32] we take $U^{2}(\mathrm{q})$ to have the form $|U(q)|^{2} \propto$ $4|q|^{2} /\left|q^{2}-\xi^{2}\right|^{2}$, where $\xi=\xi_{1}-i \xi_{2}=\left(2 \omega_{i} / c\right) \times(n-i k)$. $U^{2}($ q) respresents a skew lineshape with a peak position $q_{0}=\left(2 \omega_{i} / c\right) \times \sqrt{n^{2}+k^{2}}$.

Generally, the matrix element of the electron-photon interaction $\gamma_{\alpha \beta}(k)$ in (2) ( $\alpha$ and $\beta$ denote the polarizations for the incident and scattered lights) includes both nonresonant (intraband) and resonant (interband) transitions [1, 15, 26]. Widely used for $\omega_{i}<<E_{g}$ effectivemass approximation reduces $\gamma_{\alpha \beta}(k)$ to the Fermi surface curvature. In this case the account of the Coulomb interaction for scalar components induces in $(2) \bar{\gamma}_{\alpha \beta}(k, q, \omega)=$ $\gamma_{\alpha \beta}(k)-\left\langle\gamma_{\alpha \beta}(k, q, \omega)\right\rangle$ instead of bare $\gamma_{\alpha \beta}(k)$ [22, 24]; angular brackets denote the averaging over the Fermi surface. Indeed, the resonant terms in Raman vertex $\gamma_{\alpha \beta}(k)$ are important in metals, as it follows from the band structure calculations and was confirmed by our results [25]. The calculation of the full Raman vertex requires a lot of computations, therefore, to make them less cumbersome, we use Eq.(2) with constant $\gamma_{\alpha \beta}(k)$. This suggests the constancy of the momentum matrix elements and photon energy denominators in $\mathrm{k}$ space for the interband transitions. Since the main aim is to analyze the ERS lineshapes, this rough simplification is partly justified by the similarity of the shapes for the spectra measured in different polarization geometries. This implies a slight effect of screening on the frequency dependence of the Raman response for the only screened $A_{1 g}$ symmetry. It was shown in [14, 15] that the Coulomb interaction has to be taken into account only for the intraband processes.

At first sight, all spectra, shown in Figs.1-3, look very similar. They contain broad continua with linearly increased intensity at small frequencies and a characteristic position of maximum. The spectra measured with different excitation energies have similar forms, as it is shown for $\mathrm{W}$ in Fig.1(b), where broad peaks with the energy of maximum near $300 \mathrm{~cm}^{-1}$ are observed at the low temperature for both excitating laser lines - 514 and $633 \mathrm{~nm}$. The first-order Raman scattering by phonons for all investigated elemental cubic metals is forbidden and the upper limit of possible second-order spectra in $\mathrm{W}$ is limited by $400 \mathrm{~cm}^{-1}$. Hence, the broad spectra observed indicate their electronic origin. The continuum frequency increases to $400 \mathrm{~cm}^{-1}$ at room temperature. We calculated the ERS spectra for W using (Eq.2) with the electron-phonon constant $\lambda=2 \int d \Omega \alpha^{2} F(\Omega) / \Omega=0$. 


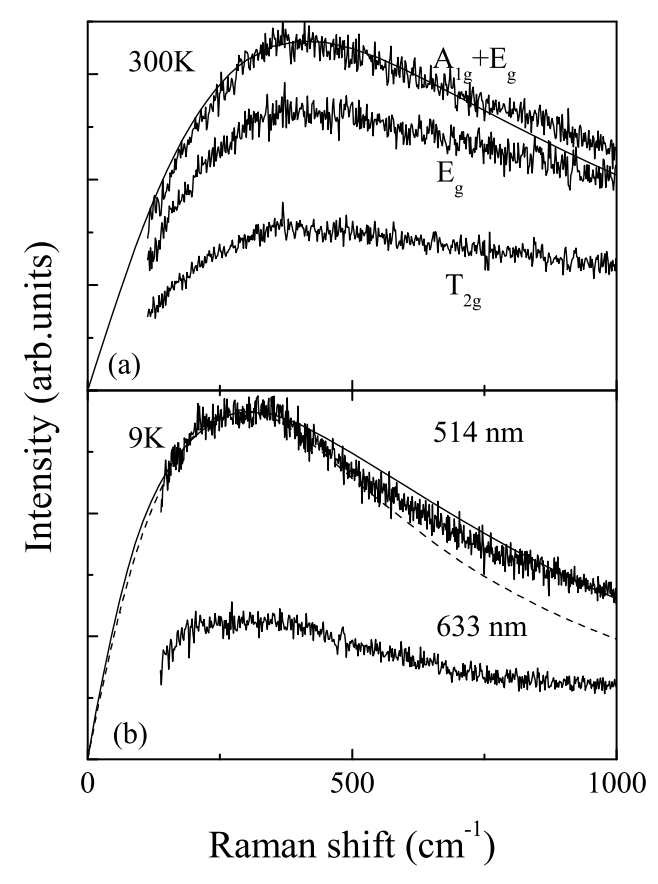

FIG. 1: Raman spectra for W, measured in different polarization geometries with $514 \mathrm{~nm}$ excitation at $300 \mathrm{~K}$-(a) and using different exciting energies at $9 \mathrm{~K}-(\mathrm{b})$. Calculated spectra are shown by solid line for $\lambda=0.13$ and dashed-for $\lambda=0$.

In such case of noninteracting electrons the spectra maximum should be found at the energy $\omega=q v_{z}$, which is determined by the average Fermi velocity $v_{z}$ and wave vector $\mathrm{q}$, corresponding to the maximum in the momentum transfer distribution $U^{2}(q)$. One can see (dashed line in Fig.1(b)) that the energy and lineshape of the calculated low-temperature continuum are very close to the experimental ones. Such a coincidence evidences that the source of the observed ERS is the intraband electronic transitions near the Fermi level within conduction bands with bare dispersion. This also confirms the conservation of the electron momentum transfer $\mathrm{q}$ in the course of the scattering process that has already been stated for Os [25, 33], where q-value variation leads to the continuum energy shift. Together with the moderate hardening of the continuum energy due to the increase of the quasiparticle damping upon increasing temperature, good agreement between experiment and calculation evidences rather weak electron interactions, i.e. small deviations from the Fermi liquid behavior. In fact, both low- and room-temperature spectra can be fitted well using $\lambda=0.13$ (Fig.1). Obviously, in the case of $\mathrm{W}$ the $q v_{z}$ term can not be neglected even at room temperature. The shapes of the observed continua are quite similar for different polarization geometries $\left(A_{1 g}+E_{g}\right), E_{g}$ and $T_{2 g}$

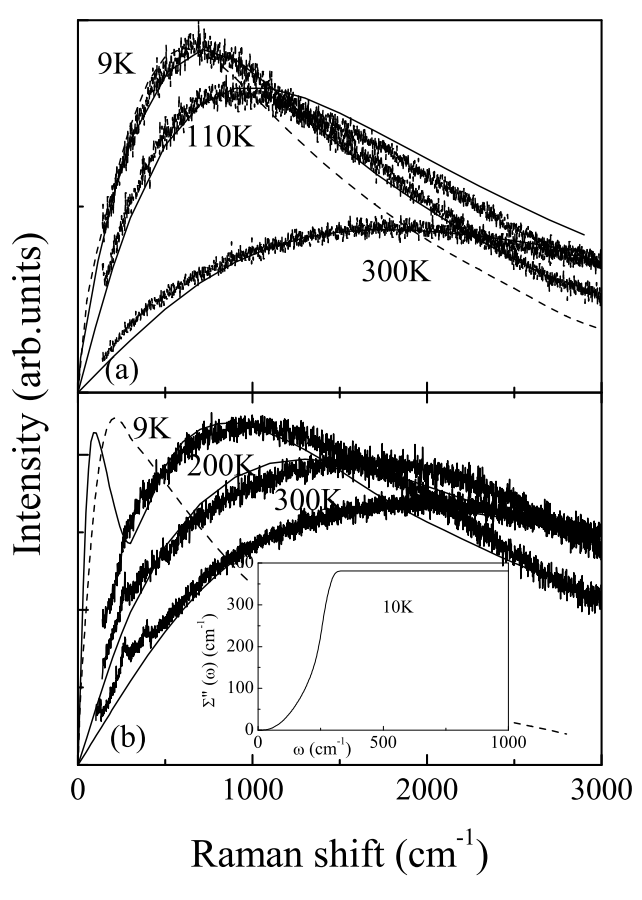

FIG. 2: Raman spectra for $\mathrm{Pb}(\mathrm{a})$ and $\mathrm{Nb}(\mathrm{b})$ at different temperatures, measured with $633 \mathrm{~nm}$ excitation. Calculated spectra are shown by solid line for $\lambda=1.04$ and dashed-for $\lambda=0$. Inset in (b) - energy dependence of the quasiparticle damping for $\mathrm{Nb}$ at $10 \mathrm{~K}$, calculated with Eq. 4 .

(Fig.1(a)) indicating the same quasiparticle lifetimes for different symmetry channels.

Next case when the $q v_{z}$ term determines the continua energies in the ERS spectra is shown in Fig.2(a). Though $\mathrm{Pb}$ is a strongly coupled superconductor, its lowtemperature ERS spectrum is very close to the calculated spectrum for noninteracting electrons (dashed line in Fig.2(a). This is due to a large value of the $q v_{z}$ term (both Fermi velocity and q are large) and the smallness of the phonon-induced electron self-energies at low temperatures. The effect of temperature on the quasiparticle damping is large: the fit of the temperature-dependent Raman spectra with Eq.2 shows the increase of the electron relaxation rate $\Gamma \approx 2 \cdot \Sigma^{\prime \prime}(\omega)$ from $150 \mathrm{~cm}^{-1}$ at $10 \mathrm{~K}$ to $1500 \mathrm{~cm}^{-1}$ at room temperature. At high temperatures this rate becomes frequency-independent and exceeds the contribution of the term with $q v_{z} \approx 600 \mathrm{~cm}^{-1}$. In this case, the $q v_{z}$ term can be neglected and a Drudelike expression for the electron response $\chi(q, \omega)$ can be used in the $q \rightarrow 0$ limit [34]:

$$
\chi(\omega) \propto N_{f} \frac{\omega \Gamma(\omega)}{\omega^{2}+\Gamma(\omega)^{2}}
$$

where $N_{f}$ is the density of states at the Fermi level. The continuum energy then should be observed at the 
frequency $\omega \approx \Gamma$, which accounts for the large shift of the ERS spectra toward higher energies when temperature increases. The experimental spectra at all temperatures were well reproduced in our calculations with $\lambda=1.04$ (Fig.2(a)). As in case of $\mathrm{W}$, only small part of the spectral intensity in $\mathrm{Pb}$ is redistributed to the highenergy range at low temperatures, indicating small deviations from the Fermi-liquid behavior. At high temperatures, a large incoherent part appears over a high-energy scale that reminds the situation in HTSCs.

In another strongly coupled superconductor $\mathrm{Nb}$ nonFermi liquid behavior can be observed already at low temperatures. While the position of maximum in the calculated ERS spectra for noninteracting electrons is 210 $\mathrm{cm}^{-1}$, the experimental value $\omega \approx 1000 \mathrm{~cm}^{-1}$, i.e. five times larger (Fig.2(b)). For Nb, both the real and imaginary parts of the electron self-energy have large values already at low temperatures. This results in the appearance of a low-frequency feature in our calculation (Fig.2(b)). This quasiparticle peak arises due to a large mass renormalization and can be still observed if the quasiparticle damping is small in this energy range, as our calculation shows (inset in Fig.2(b)). The same result was found for other strongly coupled metals Ta and V. However, we did not observe signatures of these features up to the detection limit of our spectrometer $(\approx 100$ $\left.\mathrm{cm}^{-1}\right)$. Possibly, this indicates an increase of coupling at low frequencies as compared with the calculation, which can lead to suppression of the low-frequency peaks. The large quasiparticle damping (inset in Fig.2(b)), which becomes frequency-independent at high temperatures, determines the appearance of broad relaxation peaks at higher energies at all temperatures, which were well reproduced in the calculations (Fig.2(b)). Thus, we firstly report the observation of the normal-state intraband electronic continuum in $\mathrm{Nb}$, the redistribution of which has been evidenced earlier [18]. The electron-phonon coupling constant in $\mathrm{Nb}$ was estimated to be $\lambda=1.27$.

The room temperature spectra for a number of metals are shown in Fig.3. As in the abovementioned examples the spectra contain the broad continua sometimes with superimposed peaks arising from the first- and secondorder phonon scattering. All spectra show rather strong temperature dependences which will be reported elsewhere. The comparison with the calculation helps to explain the energy positions of the experimental peaks for each metal. For example, the ERS spectra for $\mathrm{Al}$ and $\mathrm{V}$ looks very similar but their continua energies are governed by different processes. In $\mathrm{Al}$ the spectrum position is determined by the $q v_{z}$ term because its roomtemperature relaxation rate $\Gamma \approx 400 \mathrm{~cm}^{-1}$ is much lower than $\mathrm{q} v_{z} \approx 1200 \mathrm{~cm}^{-1}$. For $\mathrm{V}$ the situation is quite contrary and the spectral weight is transferred to high energies due the strong electron-phonon scattering. The frequency dependences of the ERS peaks and their temperature dependences are satisfactorily fitted by (Eq.2) and magnitudes of the electron-coupling constant $\lambda$ are estimated from these fits (Fig.3). The derived values of $\lambda$ and relaxation rates $\Gamma$ are given in Table I for all investigated metals together with available data from literature. We should note a rather good coincidence between the derived and literature $\lambda$ for strongly coupled metals (having superconducting temperatures $T_{c} \geq 1^{\circ} \mathrm{K}$ ) while for the low $T_{c}$ metals, our constants are, as a rule, smaller than the literature ones. The frequency dependence of the ERS spectra results from both the scattering processes of carriers due to interaction with phonons and Coulomb repulsion. It may be suggested that the experiment measures the effective coupling constant which is renormalized by the Coulomb interaction. It is such effective coupling constant that determines transition temperature for weakly coupled systems 35.

In summary, we presented convincing evidence for the ERS observation in metals, which is, indeed, attributed to intraband electronic excitations. An account of finite q effects when calculating the ERS spectra is important, especially for metals with the weak electron-phonon coupling even at high temperatures. In the strongly coupled systems the relaxation rate for electrons usually overcomes the $q v_{z}$ term that allows one to obtain a fair de-

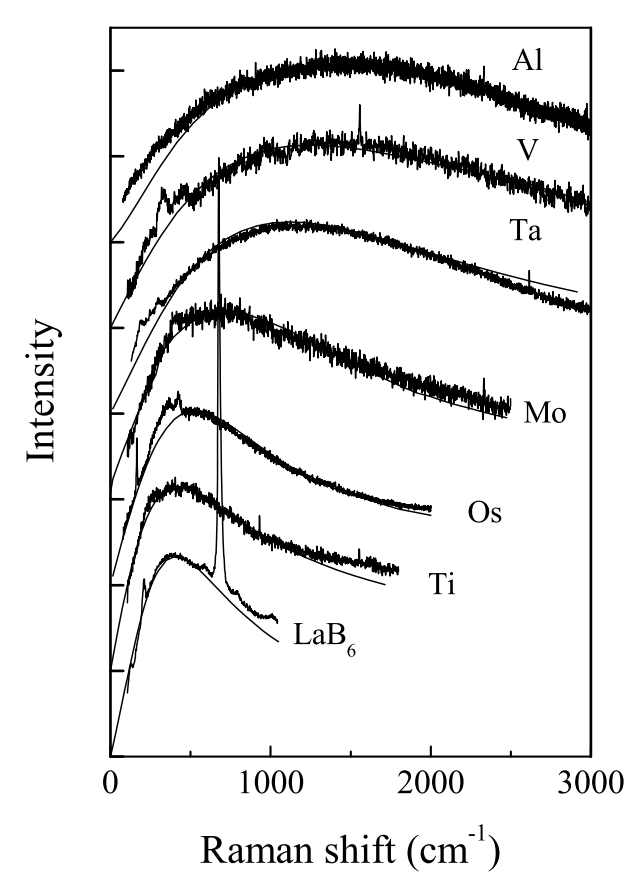

FIG. 3: Raman spectra for a number of metals taken at 300K. Narrow lines in some spectra are the first- and second-order phonon scattering. Calculated spectra are shown by solid lines. The broad bump near $2000 \mathrm{~cm}^{-1}$ in $\mathrm{La} B_{6}$, observed only with $514 \mathrm{~nm}$ excitation, is not the Raman peak. 
TABLE I: Experimental values for the electron-phonon coupling $\lambda_{\text {exp }}$ and room temperature relaxation frequency $\Gamma_{\text {exp }}$ (in $\mathrm{cm}^{-1}$ ). Literature data of the transport $\lambda_{t r}$ [36, 37], calculated by McMillan [35, 38] $\lambda_{M c M}$ and $\Gamma_{o p t}$ from optical experiments 39 41] are shown for comparison.

\begin{tabular}{llllll}
\hline \hline & $\lambda_{\exp }$ & $\lambda_{t r}$ & $\lambda_{M c M}$ & $\Gamma_{\exp }$ & $\Gamma_{\text {opt }}$ \\
$\mathrm{Al}$ & 0.26 & 0.39 & 0.38 & 375 & 557 \\
$\mathrm{Mo}$ & 0.33 & 0.32 & 0.41 & 450 & 446 \\
$\mathrm{Nb}$ & 1.15 & 1.06 & 0.82 & 1500 & 1210 \\
$\mathrm{Os}$ & 0.3 & 0.54 & 0.39 & 360 & 530 \\
$\mathrm{~Pb}$ & 1.04 & 1.48 & 1.12 & 1360 & 1625 \\
$\mathrm{Re}$ & 0.77 & 0.76 & 0.46 & 1010 & 585 \\
$\mathrm{Ta}$ & 0.83 & 0.87 & 0.65 & 1080 & 616 \\
$\mathrm{Ti}$ & 0.31 & 0.54 & 0.38 & 430 & 400 \\
$\mathrm{~V}$ & 0.87 & 1.09 & 0.6 & 1140 & 637 \\
$\mathrm{~W}$ & 0.13 & 0.26 & 0.28 & 175 & 265 \\
$\mathrm{La} B_{6}$ & 0.19 & & 0.33 & 270 & 160 \\
\hline \hline
\end{tabular}

scription using the Drude-like expression in the collision limited regime. The electron-phonon interaction becomes apparent in the ERS spectra of metals owing to transfer of the spectral intensity to the high frequency range. It is found to be the leading channel of the electron selfenergy in the investigated metals. The relaxation rates for electrons $\Gamma$ and electron-phonon coupling constants $\lambda$ are estimated from the comparison of the experimental spectra with the calculated ones. The agreement between the obtained $\lambda$ values and those available in literature is fairly good. Thus, the ERS is shown to be an alternative to the transport and optical measurements to estimate the strength of the electron-phonon interaction.

This research was partly supported by RFBR grants No. 11-02-00306 and 10-02-96011 and by UD RAS program for young scientists. Authors acknowledge A.A. Eliseev for help with low temperature measurements.

[1] T. P. Devereaux and R. Hackl, Rev. Mod. Phys. 79, 175 (2007).

[2] I. Bozovic, , D. Kirillov, A. Kapitulnik, K. Char, M. R. Hahn, M. R. Beasley, T. H. Geballe, Y. H. Kim, and A. J. Heeger, Phys. Rev. Lett. 59, 2219 (1987).

[3] Cooper, S. L., M. V. Klein, B. G. Pazol, J. P. Rice, and D. M. Ginsberg, Phys. Rev. B 37, 5920 (1988).

[4] S. L. Cooper, F. Slakey, M. V. Klein, J. P. Rice, E. D. Bukowski, and D. M. Ginsberg, Phys. Rev. B 38, 11934 (1988).

[5] R. Hackl, W. Glaser, P. Muller, D. Einzel, and K. Andres, Phys. Rev. B 38, 7133 (1988).

[6] T. Staufer, R. Hackl, and P. Muller, Solid State Commun. 75, 975 (1990).

[7] Varma, C. M., Physica C 162-164, 303, (1989).

[8] A. Virosztek, and J. Ruvalds, Phys. Rev. Lett. 67, 1657
(1991); Phys. Rev. B 45, 347 (1992)

[9] A. P. Kampf, and W. Brenig, Z. Phys. B: Condens. Matter 89, 313 (1992).

[10] T. P. Devereaux, and A. P. Kampf, Phys. Rev. B 59, 6411 (1999).

[11] E. G.Maksimov, M. L. Kulic, and O. V. Dolgov, Advances in Condensed Matter Physics 2012, ID 423725 (2010).

[12] V. N. Kostur and G. M. Eliashberg, JETP Letters, 53, 391 (1991); V. N. Kostur, Z. Phys. B: Condens. Matter 89, 149 (1992).

[13] K. Itai, Phys. Rev. B 45, 707 (1992).

[14] S. N. Rashkeev and G. Wendin, Z. Phys. B 93, 33 (1993).

[15] M. V. Klein, and S. B. Dierker, Phys. Rev. B 29, 4976 (1984).

[16] M. V.Klein, in Light Scattering in Solids III, edited by M. Cardona and G. Guntherodt Springer-Verlag, Berlin, 1982, p. 121.

[17] R. Hackl, R. Kaiser, and S. Schicktanz, J. Phys. C 16, 1729 (1983)

[18] S. B.Dierker, , M. V. Klein, G. W. Webb, and Z. Fisk, Phys. Rev. Lett. 50, 853 (1983).

[19] B. I. Verkin and B. G. Lazarev, Izv. Akad. Nauk SSSR, Ser. Fiz. 12, 598 (1948)

[20] M. S. Khakin and V. P. Bykov, Sov.Phys. JETP 3, 119 (1956).

[21] A. A. Abrikosov and L. A. Falkovski, Sov. Phys. JETP 13, 179 (1961).

[22] A. A. Abrikosov, and V. M. Genkin, Sov. Phys. JETP 38, 417 (1974).

[23] D. L. Mills, A. A. Maradudin and E. Burstein, Ann.Phys. 56, 504 (1970).

[24] I. P. Ipatova, M. I. Kaganov, and A. V. Subashiev, Sov. Phys. JETP 57, 1066 (1983).

[25] Yu. S. Ponosov, G. A. Bolotin, C. Thomsen, and M. Cardona, Phys. Status Solidi B 208, 257 (1998).

[26] L. A. Falkovskii, Sov. Phys. JETP 76, 331 (1993).

[27] E. G. Maksimov and S. V. Shulga, Solid State Commun. 97, 553 (1996).

[28] O.K. Andersen and O. Jepsen, Phys. Rev. Lett. 53, 2571 (1984).

[29] S.V. Shulga, O.V. Dolgov, and E.G. Maksimov, Phys. C 178, 266 (1991).

[30] Landolt-Bornstein, New Series III/13a (1981).

[31] Landolt-Bornstein, New Series III/15b (1985).

[32] A. Dervisch and R. Loudon, J. Phys. C 9, L669 (1976). M.M. Kirillova, L.V. Nomerovannaya, Sov. Phys. Solid State 20, 984 (1978).

[33] Yu. S. Ponosov and S. V. Streltsov, JETP Letters, 94, 437 (2011).

[34] A. Zawadowski,, and M. Cardona, Phys. Rev. B 42, 10732(1990).

[35] W. L. McMillan, Phys. Rev. 167, 331(1968).

[36] B. A. Sanborn, P. B. Allen, and D. A. Papaconstantopoulas, Phys. Rev. B40, 6037 (1989).

[37] P. B. Allen, Phys. Rev. B36, 2920 (1987).

[38] G. Schell, H. Winter, H. Rietschel, and F. Gompf, Phys. Rev. B25, 1589 (1982).

[39] M.M. Kirillova, L.V. Nomerovannaya and M. M. Noskov, Sov. Phys. Solid State 16, 1425 (1974).

[40] G. P. Motulevich, Sov. Phys. Usp. 12, 80 (1969).

[41] Shin-ichi Kimura, Takao Nanba, Satoru Kunii and Tadao Kasuya, Phys. Rev. B50, 1406 (1994). 